\title{
Machine Learning Methods for Intelligent Abnormal Brain Identification
}

\author{
Fangyuan $\mathrm{Liu}^{1}$, Siyuan $\mathrm{Lu}^{1, *}$ and Leonid Snetkov ${ }^{2}$ \\ ${ }^{1}$ School of Computer Science and Technology, Nanjing, Jiangsu 210023, China \\ ${ }^{2}$ Department of Computer Technologies and Control Systems, ITMO University, Kronverksky Prospect 197101, Russian \\ Federation \\ ${ }^{*}$ Corresponding author
}

\begin{abstract}
This survey paper describes a focused literature survey of machine learning methods in order to detect pathological brain. Based on the published time and emerging methods, this paper introduces in details the methods used in each documents. Because of the requirement to select a good approach in the process of pathological brain analysis, we compare the classification results of different methods and present a promising future.
\end{abstract}

Keywords-pathological brain detection; machine learning; intelligent algorithm; category recognition

\section{INTRODUCTION}

With the change of people's lifestyle and the rapid arrival of the population aging, brain disease now becomes a major disease that has endangered the health of the elderly [1-3]. In this case, the medical diagnostic personnel need to identify the abnormal brain timely and effectively. There are several machine-learning methods to solve the problem.

Machine learning (ML) is a significant technology that allows computers to discover hidden areas where they are not explicitly programmed [4-8]. ML can produce models faster and automatically, used to reproduce known patterns and knowledge. ML methods focus on achieving high-value predictive results and apply these results to decisions and actions. Therefore, ML is widely used in computer vision [9], biometric identification [10], medical diagnosis [11], and so on [12].

The ML methods are summarized in this paper is useful to solve the normal and abnormal brain detection problems. At the same time, we compare the evaluation results of each approach.

\section{METHODS}

Yang (2016) [13] presented a new approach respond to wavelet energy because WN shows excellent performance in practical application. The weights of SVM are optimized using BBO before classification. After that, WN and SVM are combined to classify magnetic resonance brain images automatically.

Nayak, Dash and Majhi (2016) [14] used a smart computer aided diagnosis system (CADS) to identify disease brain images. This system includes three aspects, applying 2D DWT to extract features, using PPCA to reduce features and using ADBRF to identify normal and disease brain.
Yang and Sun (2016) [15] suggested BPSO-MT to select only two features from both approximation and detail sub-bands. BPSO-MT includes two improvements, one is adding mutation operator and the other is combining with time-varying acceleration coefficient technique. To ensure the classification performance of BPSO-MT, which is compared with BPSO-M and BPSO-T in the literature.

Sun (2016) [16] used an attractive method of assisting to recognize the patterns, types and conditions of the brain. In "WE + QPSO-KSVM", after the outstanding feature extraction, the weights of the classifier are optimized effectively.

Atangana (2016) [17] proposed a novel method, stationary wavelet entropy (SWE), to extract features. Compared with the following three approaches: (i) WE; (ii) WN; (iii) DWT, the performance of SWE is best. The experimental results are $98.82 \%, 96.00 \%, 99.76 \% 98.67 \%$ for sensitivity, specificity, precision and accuracy, respectively.

Sun (2016) [18] provided a system to detect pathological brain better. The most important thing is to make two improvements to MLP. On the one hand, the best pruning technique is chosen to determine the number of hidden neurons by comparing three pruning techniques. On the other, the authors apply ARCBBO to train the biases and weights of MLP by comparing it to $\mathrm{BBO}$ and RCBBO. The accuracy of the experiment achieved $99.53 \%$.

Yang (2016) [19] described three classifiers and a comparative study on them is reported. At the first stage, applying DTCWT to transform wavelets in order to improve the weakness of DWT. Then, feature extraction is performed by VE. Meanwhile, this paper testify "DTCWT + VE + TSVM" achieved the highest accuracy compared with "DTCWT + VE + SVM" and "DTCWT + VE + TSVM".

Chen (2016) [20] developed contemporary PBDS based on single slice is the highlight in this paper. Firstly, they focus on the changes of fractal pattern and extract features using FD. Then, an improved PSO algorithm is put forward to avoid to plunging into local minimum in training phase. SLFN is applied to distinguish normal and abnormal brain images. The prospect of the overall approach is bright.

Chen, Yang and Phillips (2015) [21] proposed the following methods to identify pathological and healthy brain images: (i) WFRFT + PCA + GEPSVM; (ii) WFRFT + PCA + TSVM; (iii) WFRFT + PCA + SVM and the first one has the 
best test results. The novelty of this paper is that they create the possibility of WFRFT to extract more effective features.

Chen and Du (2017) [22] introduced a detection system based on disease brain images that includes three parts. Using WPTE to extract global features at first, using RCBBO to train the classifier in the next step and using FNN to categorize at last.

Yang (2017) [6] approved Hu moment invariants (HMI) to describe features which take the place of traditional DWT. Although the performance measure is $98.89 \%$, there are only 90 images used in this work.

Wang and Lv (2016) [23] improved particle swarm optimization (PSO) by embed predator-prey operator to train weights and biases of SLN. The experiment prediction achieved $97.02 \pm 0.33 \%$.

Jiang and Zhu (2017) [24] found pseudo Zernike moment (PZM) that represent a feature is effective to categorize. According to the paper, they not only analyzed the reason that KSVM performs better than SVM and also contrast "PZM + KSVM" with eleven previous approaches.

\section{RESULTS}

In the above approaches of this survey paper, we analyze and research the performance and disadvantages of each method in Table 1.

TABLE I. COMPARISON OF SEVERAL CONTEMPORARY METHODS

\begin{tabular}{|c|c|c|c|}
\hline Author & Method & Vulnerability & Accuracy \\
\hline Yang (2016) [13] & $\mathrm{WE}+\mathrm{BBO}-\mathrm{KSVM}$ & $\begin{array}{l}\text { Wavelet entropy has lower performance than } \\
\text { other ways. }\end{array}$ & $97.78 \%$ \\
\hline $\begin{array}{l}\text { Nayak, Dash and Majhi } \\
\text { (2016) [14] }\end{array}$ & 2D-DWT + PPCA + ADBRF & $\begin{array}{l}\text { Using PPCA to reduce features are able to } \\
\text { improve. }\end{array}$ & $99.53 \%$ \\
\hline Yang and Sun (2016) [15] & $\mathrm{WE}+\mathrm{BPSO}-\mathrm{MT}+\mathrm{PNN}$ & $\begin{array}{l}\text { Comparatively time-consuming in the } \\
\text { experiment. }\end{array}$ & $99.53 \%$ \\
\hline Sun (2016) [16] & WE + QPSO-KSVM & $\begin{array}{l}\text { not fully capture the focus and deformed } \\
\text { region }\end{array}$ & $98.22 \%$ \\
\hline Atangana (2016) [17] & SWE + RBF-KSVM & low validity during parameter optimization & $100 \%$ \\
\hline Sun (2016) [18] & $\mathrm{FRFE}+\mathrm{KC}-\mathrm{MLP}+\mathrm{ARCBBO}$ & $\begin{array}{l}\text { Compared with other approaches, the } \\
\text { sensitivity and specificity are lower. }\end{array}$ & $99.53 \%$ \\
\hline Yang (2016) [19] & DTCWT + VE + TSVM & Computation time is not optimal. & $99.57 \%$ \\
\hline Chen (2016) [20] & MBD + SLFN + PSO-TTC & Other algorithm can optimize SLFN better & $98.08 \%$ \\
\hline $\begin{array}{l}\text { Chen, Yang and Phillips } \\
\text { (2015) [21] }\end{array}$ & WFRFT + PCA + GEPSVM & Only applied to specific T2-weighted images & $99.11 \%$ \\
\hline Chen and Du (2017) [22] & $\mathrm{WPTE}+\mathrm{FNN}+\mathrm{RCBBO}$ & $\begin{array}{l}\text { The number of running times is inconsistent } \\
\text { slightly. }\end{array}$ & $99.49 \%$ \\
\hline Yang (2017) [6] & $\begin{array}{c}\text { HMI + GEPSVM and HMI + } \\
\text { TSVM }\end{array}$ & The dataset only includes 90 specific images. & $98.89 \%$ \\
\hline Wang and Lv (2016) [23] & $\mathrm{HMI}+\mathrm{SLN}+\mathrm{PP}-\mathrm{PSO}$ & The test results are unstable. & $97.02 \pm 0.33 \%$ \\
\hline Jiang and Zhu (2017) [24] & PZM + KSVM & $\begin{array}{l}\text { There are other better SVM improvements to } \\
\text { classify. }\end{array}$ & $99.45 \pm 0.38 \%$ \\
\hline
\end{tabular}

\section{CONCLUSION}

This study compared the latest machine-learning methods for abnormal brain identification. Furthermore, we look forward to make a comparison of the algorithms for detecting other human brain-related diseases, such as sickle cell disease [25], HIV disease [26], etc. Some image preprocessing methods [27-34] may be added to check the performance.

\section{ACKNOWLEDGEMENT}

This paper is supported by Research Innovation Program for College Graduates of Jiangsu Province (KYLX16_1292).

\section{REFERENCE}

[1] Brenowitz, W.D., C.D. Keene, S.E. Hawes, et al., Alzheimer's disease neuropathologic change, Lewy body disease, and vascular brain injury in clinic- and community-based samples. Neurobiology of aging, 2017. 53: pp. 83-92
[2] Lange, M., J. Mauerer, J. Schlaier, et al., Underutilization of deep brain stimulation for Parkinson's disease? A survey on possible clinical reasons. Acta Neurochirurgica, 2017. 159(5): pp. 771-778

[3] Drouet, C., O. Morel, L. Thines, et al., Brain Perfusion Before and After Surgical Treatment of Moyamoya Disease. Clinical nuclear medicine, 2017. 42(5): pp. e269-e272

[4] Yuan, T.F., Detection of subjects and brain regions related to Alzheimer's disease using 3D MRI scans based on eigenbrain and machine learning. Frontiers in Computational Neuroscience, 2015. 9, Article ID: 66

[5] Zhang, Y., Detection of Alzheimer's disease by displacement field and machine learning. PeerJ, 2015. 3, Article ID: e1251

[6] Yang, J., Pathological brain detection in MRI scanning via Hu moment invariants and machine learning. Journal of Experimental \& Theoretical Artificial Intelligence, 2017. 29(2): pp. 299-312

[7] Zhou, X.-X., Comparison of machine learning methods for stationary wavelet entropy-based multiple sclerosis detection: decision tree, k-nearest neighbors, and support vector machine. Simulation, 2016. 92(9): pp. 861-871 
[8] Hagenauer, J. and M. Helbich, A comparative study of machine learning classifiers for modeling travel mode choice. Expert Systems with Applications, 2017. 78: pp. 273-282

[9] Lemley, J., S. Bazrafkan, and P. Corcoran, Deep Learning for Consumer Devices and Services Pushing the limits for machine learning, artificial intelligence, and computer vision. IEEE Consumer Electronics Magazine, 2017. 6(2): pp. 48-56

[10] 10. Martin-Navarro, A., A. Gaudioso-Simon, J. Alvarez-Jarreta, et al., Machine learning classifier for identification of damaging missense mutations exclusive to human mitochondrial DNA-encoded polypeptides. BMC Bioinformatics, 2017. 18, Article ID: 158

[11] de Bruijne, M., Machine learning approaches in medical image analysis: From detection to diagnosis. Medical Image Analysis, 2016. 33: pp 94-97

[12] Yu, B., Machine learning and its applications. Neural Network World, 2016. 26(3): pp. 203-204

[13] Yang, G., Automated classification of brain images using wavelet-energy and biogeography-based optimization. Multimedia Tools and Applications, 2016. 75(23): pp. 15601-15617

[14] Nayak, D.R., R. Dash, and B. Majhi, Brain MR image classification using two-dimensional discrete wavelet transform and AdaBoost with random forests. Neurocomputing, 2016. 177: pp. 188-197

[15] Yang, J.F. and P. Sun, Magnetic resonance brain classification by a novel binary particle swarm optimization with mutation and time-varying acceleration coefficients. Biomedical Engineering-Biomedizinische Technik, 2016. 61(4): pp. 431-441

[16] Sun, P., Preliminary research on abnormal brain detection by wavelet-energy and quantum-behaved PSO. Technology and Health Care, 2016. 24(s2): pp. S641-S649

[17] Atangana, A. Application of stationary wavelet entropy in pathological brain detection. Multimedia Tools and Applications, 2016, DOI: 10.1007/s11042-016-3401-7 (Online).

[18] Sun, Y., A Multilayer Perceptron Based Smart Pathological Brain Detection System by Fractional Fourier Entropy. Journal of Medical Systems, 2016. 40(7), Article ID: 173

[19] Yang, M., Dual-Tree Complex Wavelet Transform and Twin Support Vector Machine for Pathological Brain Detection. Applied Sciences, 2016. 6(6), Article ID: 169

[20] Chen, X.-Q., Fractal dimension estimation for developing pathological brain detection system based on Minkowski-Bouligand method. IEEE Access, 2016. 4: pp. 5937-5947

[21] Chen, S., J.-F. Yang, and P. Phillips, Magnetic resonance brain image classification based on weighted-type fractional Fourier transform and nonparallel support vector machine. International Journal of Imaging Systems and Technology, 2015. 25(4): pp. 317-327

[22] Chen, P. and S. Du, Pathological Brain Detection via Wavelet Packet Tsallis Entropy and Real-Coded Biogeography-based Optimization. Fundamenta Informaticae, 2017. 151(1-4): pp. 275-291

[23] Wang, H. and Y. Lv Smart pathological brain detection system by predator-prey particle swarm optimization and single-hidden layer neural-network. Multimedia Tools and Applications, 2016, DOI: 10.1007/s11042-016-4242-0 (Online).

[24] Jiang, Y. and W. Zhu Exploring a smart pathological brain detection method on pseudo Zernike moment. Multimedia Tools and Applications, 2017, DOI: 10.1007/s11042-017-4703-0 (Online).

[25] LeBlanc, D., B. Towne, J. Paul, et al., Reduced Glutamate Channel Phosphorylation in Pain-Related Brain Areas in a Transgenic Model of Sickle Cell Disease. Pediatric Blood \& Cancer, 2017. 64: pp. S4-S5

[26] Lewis-de los Angeles, C.P., P.L. Williams, Y.L. Huo, et al., Lower total and regional grey matter brain volumes in youth with perinatally-acquired HIV infection: Associations with HIV disease severity, substance use, and cognition. Brain Behavior And Immunity, 2017. 62: pp. 100-109

[27] $\mathrm{Wu}, \mathrm{L}$., Improved image filter based on SPCNN. Science in China Series F-Information Sciences, 2008, 51(12): pp. 2115-2125

[28] Wu, L., Segment-based coding of color images, Science in China F edition: Information Science. 2009, 52(6): pp. 914-925
[29] Wei, G., Color Image Enhancement based on HVS and PCNN, Science China Information Sciences. 2010, 53 (10): pp. 1963-1976

[30] Dong, Z.C., A hybrid method for MRI brain image classification, Expert Systems with Applications. 2011, 38(8): pp. 10049-10053

[31] Ji, G., Effect of spider-web-plot in MR brain image classification, Pattern Recognition Letters. 2015, 62: pp. 14-16

[32] Phillips, P., Detection of Alzheimer's disease and mild cognitive impairment based on structural volumetric MR images using 3D-DWT and WTA-KSVM trained by PSOTVAC, Biomedical Signal Processing and Control. 2015, 21: pp. 58-73

[33] Chen, S., Magnetic resonance brain image classification based on weighted-type fractional Fourier transform and nonparallel support vector machine, International Journal of Imaging Systems and Technology, 2015, 24(4): pp. 317-327

[34] Peng, B., Image processing methods to elucidate spatial characteristics of retinal microglia after optic nerve transection, Scientific Reports. 2016, 6, Article ID: 21816 